\title{
GSM: gateway selection mechanism for strengthening inter-cluster coordination in cognitive radio ad hoc networks
}

\author{
Najam ul Hasan, Waleed Ejaz, Kamran Manzoor and Hyung Seok Kim
}

\begin{abstract}
In the open access spectrum paradigm, the dynamics of the available spectrum heterogeneity due to the geographical location of various cognitive radio/secondary users (SUs) and primary users (PUs) prevent the assignment of a single common control channel to every SU. Thus, depending on channel availability, SUs are forced to form clusters to solve the problem of control channel assignment. However, the sudden appearance of a PU may lead to loss of intra-cluster or inter-cluster connectivity among SUs. This problem can be resolved by improving the cluster formation process and selecting suitable gateway nodes for inter-cluster coordination. In this paper, our goal is to improve inter-cluster connectivity by selecting more appropriate gateway nodes for inter-cluster coordination. We therefore propose an inter-cluster gateway node selection mechanism that strengthens inter-cluster coordination. Our scheme shows significant improvement in terms of inter-cluster connectivity compared with related work.
\end{abstract}

Keywords: Cognitive radio; Control channel; Gateway selection

\section{Introduction}

Cognitive radio (CR) networks have gained much attention in the research community in the last decade and have emerged as a promising approach to solving the issues of spectrum scarcity [1]. CR is the key technology underlying the dynamic spectrum access (DSA) paradigm. In DSA, CR networks are overlaid over the licensed networks, also known as primary networks, for opportunistic spectrum access [2]. Consequently, in a network that utilizes DSA, users are classified as primary if they are licensed to operate in a particular frequency band; and secondary if otherwise. Secondary users (SUs) can operate in the vacant portion of the licensed band only if they do not interfere with the primary users (PUs) [3]. When a cognitive radio ad hoc network (CRAHN) is set up, SUs need to cooperate by exchanging control information to achieve reliability in seeking vacant frequency bands. As a result, the establishment of a control channel is essential for exchanging control information [1-3]. A comprehensive survey of control channels in CR networks is given in [4].

* Correspondence: hyungkim@sejong.edu

Department of Information and Communication Engineering, Sejong University, Gunja-Dong 98, Gwangjin-gu, Seoul 143-747, Republic of Korea
When setting up a CRAHN, a control channel is essential in the carrying out of functions such as spectrum sensing, sharing, decision, and mobility as all these require extensive control information exchange [1]. Several techniques have been proposed in the literature pertaining to the design of control channels for CRAHNs. Most of the proposed techniques can be divided into two categories: (1) dedicated common control channels and (2) group/cluster-based control channels. Using a dedicated common control channel simplifies the process of coordination among SUs. However, reliance on a dedicated common control channel results in several limitations. Firstly, there is a single point of failure; for example, a dedicated common control channel can result in loss of connectivity among SUs in cases of saturation or jamming. Secondly, in the case of CRAHNs, the dynamically changing availability of the spectrum in the temporal and spatial domain makes it difficult to maintain a dedicated common control channel that is globally available.

Two major challenges arise in the assignment of a dedicated control channel for CRAHNs, namely, PU activity and spectrum heterogeneity. With regard to PU activity, in a CRAHN, a PU has higher priority than an SU. If an $\mathrm{SU}$ is operating on a particular frequency band and a PU 
comes back to that frequency band, the SU has to vacate that channel in favor of the PU and migrate to some other unused frequency band. Thus, in environments where PU activity is not known, it is impossible to guarantee that the particular frequency band of the designated control channel will always remain available in the presence of PU activity. With regard to spectrum heterogeneity, every SU observes a different set of available channels depending on its geographical location, each of which is a subset of the set of all the licensed channels. Due to this spectrum heterogeneity, very rarely can a channel commonly available to all SUs at a particular instance be found. To account for PU dynamics and spectrum heterogeneity issues, several researchers have proposed cluster-based approaches for control channel design of CRAHNs [5-8]. In cluster-based control channel schemes for CRAHNs, a neighbor discovery (rendezvous) process is performed to set up an initial network topology. Based on the information exchanged during the rendezvous process, the nodes are partitioned into clusters depending on highly correlating spectrum opportunities. A comprehensive survey on rendezvous process for cognitive radio is presented [9]. After clustering, one or more channels that are common to nodes within each cluster are designated as the control channels.

In [9], Zhao et al. proposed a distributed cluster-based approach for control channel design in CRAHNs. This scheme partitions the nodes into clusters, with the largest possible number of nodes in each cluster. This method reduces the number of distinct frequencies used for control channels thereby incurring less cluster management overhead. However, the scheme requires frequent re-clustering in response to PU activity. Another similar approach for clustering CR networks is proposed in [10].

To avoid frequent re-clustering in response to PU activity and spectrum heterogeneity, the concept of backup control channels has been introduced. Bahl et al. [11] proposed a control channel scheme for centralized CR networks in which one main channel and one backup channel are designated as control channels. The main and backup channels are then interchanged according to the spectrum dynamics incurred due to PU activity. In [12], Liu et al. extended this concept of backup control channel in CRAHNs by proposing a cluster-based control channel allocation scheme called spectrum opportunity-based clustering (SOC). Instead of making the cluster with the largest number of nodes as proposed in [13] or with just one backup channel as proposed in [11], they proposed that each cluster be formed in such a way that the product of the number of common channels among cluster members and the number of cluster members in a cluster are maximized. In other words, clusters are formed in such a way that a balance is created between the cluster size and the number of common channels.
By maximizing the number of common channels among the nodes within a cluster, intra-cluster connectivity is strengthened and frequent re-clustering averted. However, the overall connectivity of a cluster-based CRAHN includes intra-cluster as well as inter-cluster connectivity. Thus, in this study, our focus is on inter-cluster connectivity, and we carry forward and enhance the concept introduced by Liu et al. [12] to improve inter-cluster connectivity. The inter-cluster connectivity is strengthened by designating more channels as control information between adjacent clusters.

Generally, a clustered ad hoc network consists of three kinds of nodes: cluster head nodes, gateway nodes, and ordinary nodes. Cluster head nodes are vested with the responsibility of managing the cluster. For example, in the case of a cluster-based control channel design for a CRAHN, the cluster head node is responsible for allocating a set of control channels out of the set of channels available to every member of that cluster. The coordination between two adjacent clusters is conducted through the gateway nodes. A node of a cluster can act as a gateway only if it has a neighboring node as well as a channel in common with the designated control channel of its neighboring cluster. All nodes other than the cluster head node and gateway node are ordinary nodes. Both gateway nodes and ordinary nodes are also known as cluster members and are managed by the cluster head node.

As mentioned earlier, in a cluster-based CRAHN, the gateway node is responsible for coordination between adjacent clusters. It addition, it is well known that inter-cluster connectivity becomes stronger the more channels there are between adjacent clusters in the case of CRAHNs. There can be nodes in a cluster that have one or more channels in common with the set of channels common among nodes in the adjacent cluster. Any of these nodes can be a gateway node candidate. However, the node with the maximum number of channels in common with the control channels in the adjacent cluster is the most suitable option because selecting such a node as a gateway strengthens the coordination with the adjacent clusters. Therefore, in this paper, we propose a mechanism for selecting gateway nodes and analyze its impact on the connectivity of CRAHNs.

The remainder of this paper is organized as follows: section 2 presents the system model used for our simulated environment. Section 3 discusses our proposed framework and describes the algorithm proposed for the selection of the gateway node for inter-cluster coordination in CRAHNs. Section 4 shows the impact of variations in SU and PU node densities on the proposed algorithm by presenting simulation results. Finally, section 5 concludes the paper. 


\section{Problem statement and system model}

\subsection{Problem statement}

Consider a geographical region in which a specific band is shared between PUs and SUs operating in that area. It is assumed that the spectrum is divided into equally spaced portions known as channels. The spectrum sharing between PUs and SUs is vertical. Thus, an SU has to ensure that no PU is transmitting on a particular channel before accessing it. To achieve this, each SU senses the spectrum after a specific interval and comes up with a set of channels $C_{i}$ available for its communication. Initially, each SU discovers its neighbors and exchanges the list of channels with them. Two nodes are neighbors if and only if both of them are within communication range of each other and they have at least one channel in common with each other. This is typically achieved during neighbor discovery phase. During this phase, all SUs obtain a list of idle channels from the local spectrum sensing observation, follow a hopping sequence, and hop-over-idle channels. Two neighboring nodes discover each other and establish a link by exchanging beacons when they hop to the same channel. This is how the initial network is formed in the absence of any predefined control channel. Based on the received information, a distributed clustering mechanism proposed by Liu et al. in [12] is employed to partition the SUs into clusters, and a cluster head is chosen for each cluster. Each cluster has a set of channels common to all the cluster members, known as the intracluster common control channel. To perform inter-cluster control information exchange, each cluster head chooses a gateway for each neighboring cluster. The common channels between the gateway and the neighboring cluster are known as inter-cluster common control channels. The goal of this study is to propose a mechanism for gateway selection in such a way that the number of inter-cluster common control channels is maximized.

For the sake of clarity, the problem mentioned above is illustrated in Figure 1 in the form of a graph, where connectivity of each neighboring pair is shown with the help of a solid line. After the neighbor-discovery process, using a distributed clustering mechanism, the SUs are partitioned into two clusters, as shown in Figure 1a. Nodes $\mathrm{A}$ and $\mathrm{E}$ are nominated as cluster heads of the cluster, as depicted in Figure 1b. The clusters are named $\Phi_{\mathrm{A}}$ and $\Phi_{\mathrm{E}}$, with the subscripts denoting the cluster heads. $Z_{\mathrm{A}}$ and $Z_{\mathrm{E}}$ are the lists of control channels for intra-cluster coordination in clusters $\Phi_{\mathrm{A}}$ and $\Phi_{\mathrm{E}}$, respectively. $C_{\mathrm{D}}$ and $C_{\mathrm{G}}$ indicate the lists of channels sensed idle by nodes D and $G$. In this example, $C_{\mathrm{D}}$ is $\{1,2,3,5,7\}$ and $C_{\mathrm{G}}$ is $\{1,2,3,4,8\}$. Only nodes D and $\mathrm{G}$ of cluster $\Phi_{\mathrm{A}}$ have neighbors in adjacent cluster $\Phi_{\mathrm{E}}$. Therefore, both nodes D and G are candidates for selection as a gateway node. This problem is referred to as the gateway node selection problem for inter-cluster coordination in CRAHNs. It is essential that there be a mechanism for selecting the node with the maximum number of channels in common with the set of selected channels for the adjacent cluster.

\subsection{System model}

As a system model, we consider a cognitive radio network that coexists with one or more primary networks in the same geographical region. The PUs can operate only in the licensed spectrum, which is divided into $T$ orthogonal frequency channels. For the sake of simplicity, it is assumed that each channel is characterized by the same attributes such as bandwidth, bit error rate, and so on. There are $n$ SUs randomly distributed in the region. The transmission range of each radio, whether it is SU or PU, is assumed to be about $\gamma$ meters. Each SU can listen to other SUs only within this transmission range.

Each SU employs a spectrum sensing technique, such as cyclostationary feature detection or energy detection, to search for the idle channels available for their usage. A comprehensive survey on spectrum sensing techniques including energy detection and cyclostationary is presented in [14]. After sensing, each ith SU has a list of available channels denoted $C_{i}$. Two SUs are called neighbors if they are within the transmission range of each other and they have at least one channel in common. Each SU discovers its neighboring SUs. The list of the neighbors of the SU is denoted $N_{i}$.

The notations used in this paper are listed in Table 1.

After neighbor discovery, each SU participates in a process of cluster formation and inter-cluster connection based on the common channels among the nodes. The cluster formation and inter-cluster connection are performed in a distributed manner, based on neighbor information. A cluster formation problem in a CR network is formally related with four items: cluster head, cluster members, common channels between the cluster members, and the cluster gateway used for inter-cluster coordination.

This paper addresses the problem of cluster gateway selection for inter-cluster coordination. Any member of a cluster that has a single neighbor node and channel in common with the set of common channels of any other adjacent cluster can be a candidate for selection as a gateway node. In this paper, our aim is to devise a mechanism for selecting a more suitable gateway node such that inter-cluster connectivity is stronger against $\mathrm{PU}$ interference. PU activity prediction is quite difficult in real time, so the goal of selecting the gateway node is to establish as many common channels between neighboring clusters as possible. If the chosen gateway has a large number of channels in common with the designated control channels for the neighboring cluster, in case a channel used for inter-cluster communication 

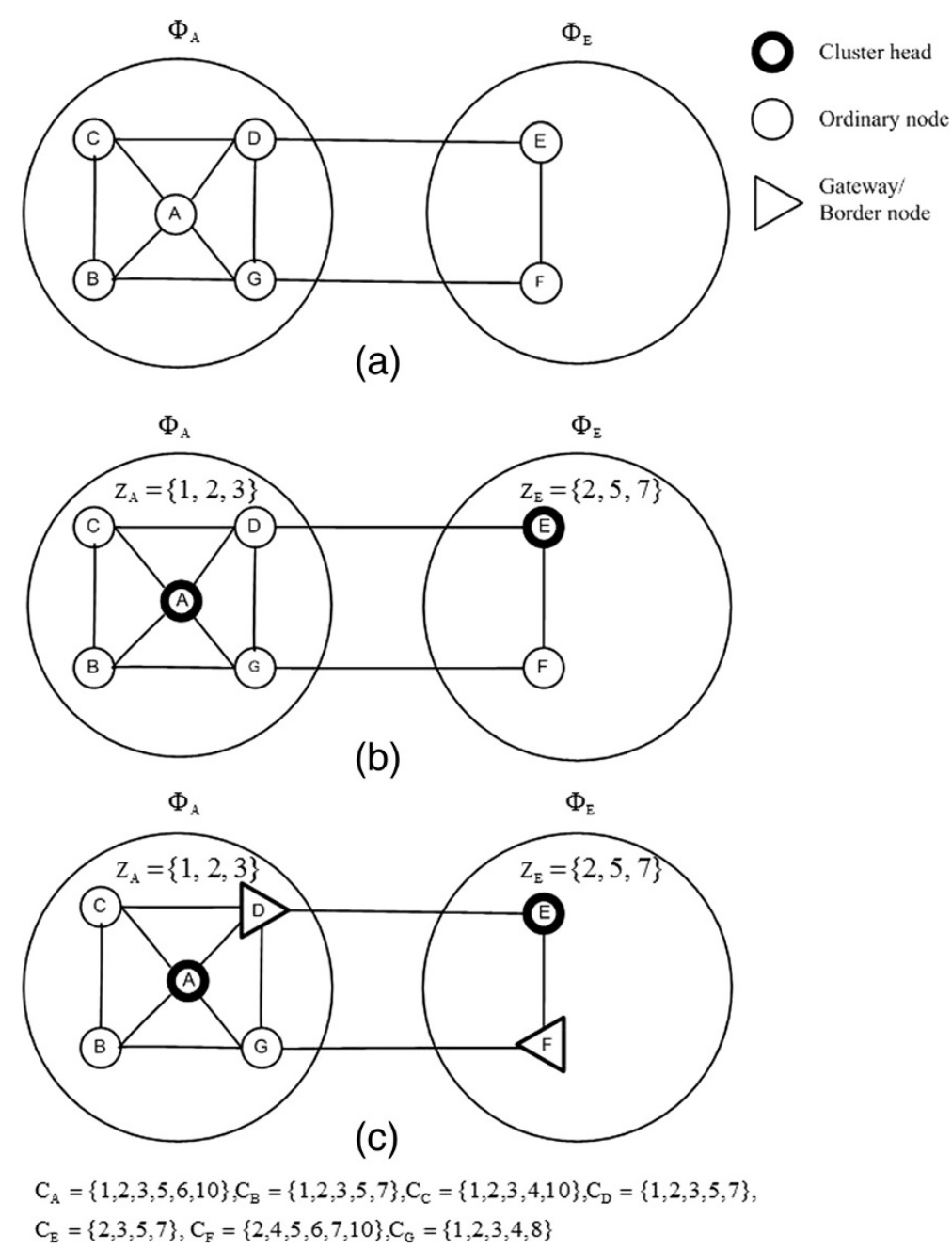

Figure 1 Clustering of connected graphs of seven SU nodes, cluster head nomination, and gateway node election. (a) An example of clustering of connected graphs of seven SU nodes in a CRAHN; (b) Cluster head nomination for each cluster; and (c) Gateway node election for each cluster.

gets occupied by a PU, the inter-cluster link can still use some other backup channels on the same gateway.

\section{Proposed framework}

To account for the space and time availability of the spectrum for control information exchange, the CRAHN is partitioned into clusters. To ensure availability of connectivity in the CRAHN, adjacent clusters must be connected to each other. To strengthen the connectivity among the clusters, we propose a gateway node selection mechanism for CRAHNs. The entire process of establishing control channels for a CRAHN can be broken down into three phases: (1) neighbor discovery, (2) cluster formation, and (3) gateway node selection. In the neighbor discovery phase, each SU discovers its neighbors by listening to different channels; however, the details of the neighbor discovery process are beyond the scope of this paper.

\subsection{Cluster formation}

To ensure that a large set of common idle channels is in each cluster and provide a graceful trade-off between the cluster size and cluster-based common idle channels, Liu et al. [12] formulated the clustering problem using a bipartite graph. In this study, we incorporate the same methodology for cluster formation. Despite of employing the same clustering as [12], proposed gateway selection mechanism (GSM) can work independent of underlying clustering scheme. However, the performance of GSM is still dependent on the underlying clustering technique. SUs are partitioned into clusters based on the set of idle channels that are common to all cluster members. The main goal of this cluster formation methodology is to cluster SUs in such a way that there is a large set of common idle channels between the cluster members. The benefit associated with this sort of clustering is that 


\section{Table 1 Nomenclature}

\begin{tabular}{|c|c|}
\hline Notation & Description \\
\hline$\Phi_{i}$ & Cluster with the ith node as the cluster head. \\
\hline$T$ & Total number of channels in the network. \\
\hline$n$ & Number of SUs. \\
\hline$C_{i}$ & List of idle channels sensed by the ith SU. \\
\hline$N_{i}$ & List of neighbors of the ith SU. \\
\hline r & Transmission range of SU or PU. \\
\hline$G_{i}\left(S_{1}, S_{2}, E\right)$ & Undirected bipartite graph at the ith SU. \\
\hline$S_{1}$ & Set of vertices including the ith $\mathrm{SU}$ and its neighbors $\mathrm{N}_{i}$. \\
\hline$S_{2}$ & $\begin{array}{c}\text { Set of vertices including a list of channels } C_{i} \text { available } \\
\text { with the ith SU. }\end{array}$ \\
\hline$X_{i}$ & $\begin{array}{l}\text { Nodes in the chosen maximum edge biclique graph } \\
\text { of the ith SU. }\end{array}$ \\
\hline$Y_{i}$ & $\begin{array}{c}\text { Channels in the chosen maximum edge biclique } \\
\text { graph of the ith SU. }\end{array}$ \\
\hline$Q\left(X_{i}, Y_{i}\right)$ & $\begin{array}{c}\text { Sub-graph of graph } G \text {, known as a multiple-edge } \\
\text { biclique graph. }\end{array}$ \\
\hline$Z_{m}$ & Common control channels assigned to the $m$ th cluster \\
\hline F & Number of elements in $X_{i}$. \\
\hline K & Number of elements in $Y_{i}$. \\
\hline$w_{i}$ & $\begin{array}{l}\text { Weight assigned to the ith } \mathrm{SU} \text { based on the } \\
\text { product of } X_{i} \text { and } Y_{i} \text {. }\end{array}$ \\
\hline$d_{i m}$ & $\begin{array}{l}\text { Degree of connectivity of an ith node to its neighbor } \\
\text { node belonging to cluster } m \text {, i.e., the intersection of } \\
C_{i} \text { and a set of idle channels chosen for cluster } m \text {. }\end{array}$ \\
\hline$\sigma$ & PU arrival rate; refers to how often a PU occupies a channel \\
\hline$a$ & $\begin{array}{l}\text { Average number of intra-cluster control channels } \\
\text { per cluster in the entire CRAHN. }\end{array}$ \\
\hline$\beta$ & $\begin{array}{c}\text { Average number of inter-cluster control channels } \\
\text { per cluster in the entire CRAHN. }\end{array}$ \\
\hline
\end{tabular}

a cluster has more options for choosing a control channel. As a result, the control channel can migrate to another channel without performing clustering again, even if the current one becomes occupied by a PU.

Following neighbor discovery and the initial exchange of the list of idle channels $C$, each $\mathrm{SU}_{i}$ becomes aware of its list of one-hop neighbors, denoted $N_{i}$, and their channel list $C_{j}$, where $S U_{j} \in N_{i}$. The cluster formation procedure consists of the following steps:

\subsubsection{Procedure: cluster formation mechanism}

Step 1: Every $i$ th SU broadcasts its set of idle channels $C_{i}$. Step 2: Every $i$ th SU constructs a bipartite graph based on $N_{i}, C_{i}$, and $C_{j}$ the list of channels of neighbors $\mathrm{SU}_{j}$.

Step 3: Every $i$ th $S U$ extracts the maximum edge biclique graph $Q\left(X_{i}, Y_{i}\right)$, as will be explained later in this section. The product of the number of nodes and channels in the chosen biclique graph represents the weight $w_{i}$ of the $i$ th SU.
Step 4: Every $i$ th SU broadcasts $w_{i}, X_{i}$, and $Y_{i}$ to its neighboring SUs.

Step 5: If $w_{i}>w_{j}, \forall j \in N_{i}$, the $i$ th $\mathrm{SU}$ is declared a cluster head; otherwise, it joins the cluster head with the greatest weight $w_{j}$ among its neighbors $N_{i}$.

\subsection{Construction of bipartite graph and extraction of maximum edge biclique graph}

After exchanging information between neighboring SUs, this information can be represented in the form of a bipartite graph. A graph $G(V, E)$ is called bipartite if the set of vertices $V$ can be partitioned into two disjoint sets $S_{1}$ and $S_{2}$ with $S_{1} \cup S_{2}=V$ such that every edge $E$ connects a vertex in $S_{1}$ to a vertex in $S_{2} . G_{i}\left(S_{1}, S_{2}, E\right)$ denotes the bipartite graph for the $i$ th node, where the set $S_{1}$ consists of $S U_{i}$ and its neighbors $N_{i}$ and the set $S_{2}$ consists of the list of idle channels $C_{i}$. An edge $(x, y)$ exists between vertices such that $x \in S_{1}$ and $y \in S_{2}$ if $y \in C_{x}$, i.e., channel $y$ is in list $C_{x}$. Figure 2a depicts the bipartite graph constructed for node $\mathrm{A}$ of the graph shown in Figure 1a. A bipartite graph $Q(V=X \cup Y, E)$ is termed biclique if for each $x \in X$ and $y \in Y$, there exists an edge between $x$ and $y$. In this study, we are interested in extracting the biclique graph that has the maximum product of cluster size and the set of channels common among cluster members. Figure $2 \mathrm{~b}$ depicts the maximum edge biclique graph for node $\mathrm{A}$.

The basic aim of our clustering scheme is to ensure that a large set of idle channels are available to the cluster members. To attain this goal, we need to cluster in such a way that the product of the number of nodes and number of channels common to each member of the cluster is maximized. The sub-graph meeting this criterion is called the maximum edge biclique graph. The algorithm for extracting this maximum edge biclique graph is presented below.

Algorithm 1 computes the maximum edge biclique graph for the $i$ th SU. In each iteration, one SU is examined. The vector I holds the indices of the SU that have already been examined; while $Y_{i}$ stores the channels that are common to SUs in I. Initially, I is empty and $Y_{i}$ contains the list of channels for the $i$ th $\mathrm{SU}$ on which the algorithm is running. The SU node $k$ to be examined in each iteration is the one whose channel list $C_{k}$ has the maximum overlap with $Y_{i}$, i.e., the number of elements in $R=Y_{i} \cap C_{k}$. Then, the examined $S U_{k}$ is removed from $S_{1}$ and the above process is repeated until either $S_{1}$ becomes empty, meaning that $M$ goes to zero or $R$ becomes null. Finally, the biclique graph $Q$ with the highest product of the number of SU nodes and the number of common control channels is eventually obtained. The outcome is a set of nodes $X_{i}$ with a set of common channels $Y_{i} . Y_{i}$ is obtained by taking the intersection of channels available to every element of $X_{i}$. The nodes having the same biclique graph form a cluster with the 
cluster head having the highest weight among all other nodes and $Y_{i}$ is declared as the set of control channels for that cluster, denoted $Z$.

\subsection{Gateway node selection mechanism (GSM)}

After clustering, the next step is to choose the gateway node for each cluster. In a cluster-based CRAHN, two clusters can access each other through different nodes and so there arises the problem of selection of the node more suitable to function as the gateway node between them. It is the responsibility of the cluster head to choose the gateway node for the adjacent clusters. The main goal of choosing a gateway and designing the control channels for a CRAHN is that the connectivity between the adjacent

\section{Algorithm 1: Extracting a maximum edge biclique graph from a bipartite graph}

1. Input: $G_{i}\left(S_{1}, S_{2}, E\right)$ Bipartite graph for the $i$ th SU.

2. Output: $Q\left(X_{i}, Y_{i}\right) \quad$ Maximum edge biclique graph for the $i$ th $\mathrm{SU}$

$$
w_{i} \quad \text { Weight of } i \text { th SU, i.e., the product of } X_{i} \text { and } Y_{i}
$$

3. $Y_{i} \leftarrow C_{i}$

4. for $j=1$ to $M \quad M$ is the number of elements in set $S_{1}$

5. Find $k$, where $S U_{k} \in \mathrm{S}_{1}$ and the number of elements in $R=Y_{i} \cap C_{k}$ is the maximum.

6. $\quad$ if $R=\varphi$

7. break

8. else

9.

10.

$$
\mathbf{I}_{i}[j]=k
$$

0. $\quad S_{1} \leftarrow S_{1}-S U_{k}, X_{i} \leftarrow X_{i} \cup S U_{k}, Y_{i} \leftarrow Y_{i} \cap C_{k}$

11. $P_{i}[j]=F \times K$, where $F$ is the number of elements in $X_{i}$ and $K$ is the number of elements in $Y_{i}$.

12. end if

13. end for

14. Find $j^{*}=\arg \max _{j} P_{i}[j]$

15. Return $w_{i}=P_{i}\left[j^{*}\right]$

16. Return $Q\left(X_{i}, Y_{i}\right)$, where $X_{i}=\left\{S U_{I_{i}[1], \ldots S U_{I_{i}\left[j^{*}\right]}}\right\} ; Y_{i}=\bigcap_{p=1}^{p=j^{*}} S U_{I_{p[k]}}$ 


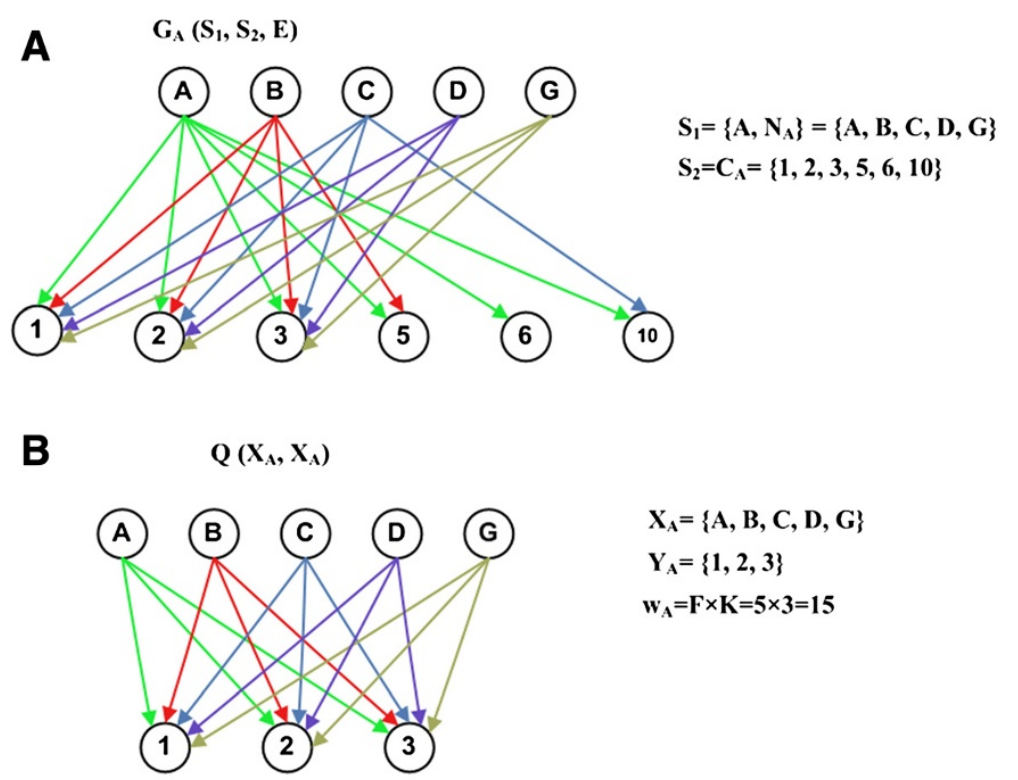

Figure 2 Bipartite graph for node A (a) and maximum edge biclique graph for node A (b).

clusters is strengthened. To strengthen the inter-cluster connectivity, there should be a large set of channels for inter-cluster communication. Therefore, the node with more channels in common with the adjacent cluster should be selected as the gateway node.

In this study, we propose a mechanism for selecting gateway nodes on the basis of degree of connectivity $d_{i m}$, where $i$ denotes a particular node and $m$ is the label of a particular cluster. The degree of connectivity of node $i$ with a particular cluster $m$ can be mathematically defined as

$$
d_{i m}=C_{i} \cap Z_{m}
$$

where $C_{i}$ is the channel list of $S U_{i}$ and $Z_{m}$ is the set of channels common to the members of the $m$ th cluster. The following steps are involved in the selection of a gateway/ border node at a particular cluster head:

\subsubsection{Procedure: gateway/border node selection}

Step 1: Each $i$ th SU broadcasts its cluster identifier and the list of control channels $Y_{\mathrm{i}}$ used in its cluster to its neighbors.

Step 2: Each $i$ th SU computes the degree of connectivity $d_{i m}$ with its neighboring clusters.

Step 3: Each $i$ th SU sends all the computed degrees of connectivity to its cluster head.

Step 4: For each neighboring $m$ th cluster, the cluster head nominates node as a gateway node that has the highest degree of connectivity and lowest id.
In the above procedure, the identifier assigned to the cluster is based on the cluster head identifier. For example, the graph in Figure 1a is clustered into two clusters labeled $\Phi_{\mathrm{A}}$ and $\Phi_{\mathrm{E}}$, where the subscripts represent the cluster heads. $Z_{\mathrm{A}}$ and $Z_{\mathrm{E}}$ are the idle channels common to the cluster members of clusters $\Phi_{\mathrm{A}}$ and $\Phi_{\mathrm{E}}$, respectively. The next step is for the cluster head A to choose the gateway node from among the members of its cluster. The cluster members $\{\mathrm{D}, \mathrm{G}\}$ of cluster $\Phi_{\mathrm{A}}$ having neighbors in $\Phi_{\mathrm{E}}$ compute their degrees of connectivity $d_{\mathrm{DE}}$ and $d_{\mathrm{GE}}$ by using Equation 1 as follows:

$$
d_{\mathrm{DE}}=C_{\mathrm{D}} \cap Z_{\mathrm{E}}=\{1,2,3,5,7\} \cap\{2,5,7\}=\{2,5,7\}
$$

$$
d_{\mathrm{GE}}=C_{\mathrm{G}} \cap Z_{\mathrm{E}}=\{1,2,3,4,8\} \cap\{2,5,7\}=\{2\} .
$$

After computing the degrees of connectivity, both nodes $\mathrm{D}$ and $\mathrm{G}$ send their degrees of connectivity $d_{\mathrm{DE}}$ and $d_{\mathrm{GE}}$ to their cluster head A. Node A then determines the node $i$ with the maximum degree of connectivity, $\operatorname{argmax}_{\mathrm{i}}\left[d_{i m}\right]$. In the above example, node D has a higher degree of connectivity than $\mathrm{G}$ because it has three channels in common with the common channels of $\Phi_{\mathrm{E}}$; thus, D is chosen as a gateway node for inter-cluster coordination between $\Phi_{\mathrm{A}}$ 
and $\Phi_{\mathrm{E}}$, as shown in Figure 1c. The algorithm for selecting the gateway node is as follows:

When a cluster head nominates the gateway for the $m$ th cluster, two possible cases can be observed:

Case 1 . There exists a valid gateway node: in cases where there are one or more nodes that have one or more number channel in common with the control channel nominated for the $m$ th cluster, one or more valid candidates can be nominated as a gateway node. In such a case, after receiving the number of channels in common between the valid gateway candidate and control channel nominated for the $m$ th cluster (also mentioned earlier as the degree of the node), the cluster head nominates the node with the greatest degree as the gateway for the $m$ th cluster.

Case 2. There exists no valid gateway node: in case that there exists no node that has any channel in common with the control channel nominated for the $m$ th cluster, the $m$ th cluster will not be considered a neighboring cluster; hence, no valid gateway exists in this scenario. Instead, the control messages between these clusters will be exchanged via another cluster. The number of hops between two clusters depends on the number of clusters. If the number of clusters is less, less number of hops will be required and vice versa. The number of clusters depends on the size of clustering, which is based on the adopted clustering mechanism. However, in case of an isolated cluster where no inter-cluster link of a cluster exists with any other cluster, the proposed GSM will not be helpful.

\section{Performance evaluation}

To evaluate the performance of proposed GSM scheme, we did a comparison with the existing SOC approach and a modified version of GSM named as GSM'. In

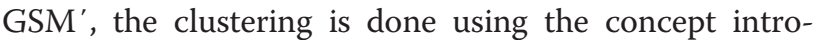
duced by [11]. In this clustering, there are one main and one backup channel in each cluster, and all the nodes which are in the communication range and have two common channels should be part of one cluster. The metrics used for the comparison were (1) average number of intra-cluster channels per cluster, denoted $\alpha$, and (2) average number of inter-cluster channels between adjacent clusters, denoted $\beta$. The intra-cluster channels are the channels available for use as control channels for local coordination within a cluster. Intercluster channels are channels available for use by a gateway for coordination with adjacent clusters. We examined the effect of varying the number of SUs and PUs in a simulation.

Let the available spectrum be divided into $T=30$ channels. It is assumed that each radio can listen to other radios within a transmission range of $x=40 \mathrm{~m}$ and the total area over which this CRAHN is set up is $100 \times 100 \mathrm{~m}$. The entire area is divided into a grid consisting of 100 equal elements. The SUs and PUs available in the network are randomly distributed in the region. Any two SUs lying in the same or adjacent grid elements have at least $70 \%$ of the available channels in common. The parameter $\sigma$ is used to denote the PU arrival rate, which indicates how often a PU occupies a channel. The value of $\sigma$ is taken to be 0.1 unless otherwise specified.

\section{Algorithm 2: Gateway node selection by cluster head A}

1. Input: $d_{i m} \quad d_{i m}$ denotes the degree of connectivity of the $i$ th node with cluster $m$

where $i$ represents an $\mathrm{SU}$ that has neighboring $\mathrm{SUs}$ belonging to another cluster.

2. Output: $g_{m} \quad$ gateway node for cluster $m$

4. for $m=1$ to $u u$ is the number of neighbor clusters

5. Find $g_{m}=\min _{i}\left\{\arg \max _{i}\left[d_{i m}\right]\right\}, \forall i \in C_{A}$

6. $\quad$ Return $g_{m}$ and declare it the gateway node selected by cluster head A for cluster $m$.

7. end for 


\subsection{Effect of variation in SU density}

Figure 3, graphically illustrates the impact of the number of SUs on the average number of clusters. As mentioned earlier in section 2, the same approach is used for clustering as used in SOC. Therefore, the same average number of clusters is formed in both the proposed GSM approach and the SOC, as depicted in Figure 3. However, in GSM', the number of clusters is less than GSM or SOC. In SOC, the objective is to maximize the product of the number of nodes and the number of common channels in each cluster. On the other hand, in GSM', the objective is to maximize the number of nodes, having just two channels in common with each other. Hence, in GSM', relatively large clusters will be formed than SOC. This will lead to reducing average number of formed clusters in GSM'. When SU density is low, most of the SUs may be disconnected from each other and more clusters are formed. When more SUs join the network, however, the average number of clusters starts to decrease because more SUs find others that are close. However, the average number of clusters starts to increase once again after a certain number of SUs have joined the network. As shown in Figure 3, this occurs after 700 SUs have joined the network.

Figure 4 illustrates the impact of the number of SUs on the average number of control channels per cluster, denoted by $\alpha$. The performance of both GSM and SOC is the same in terms of the value of $\alpha$. However, the value for GSM' $^{\prime}$ is quite less than that for GSM because GSM' tries to maximize nodes in the cluster rather than common channels, therefore, all the nodes within communication range having at least two channels in common are included in one cluster. Therefore, the value of common channels for GSM' is greater than 2 but not comparable with GSM. The value of $\alpha$ decreases as the number of SUs increases.

Figure 5 shows the effect of increasing the number of SUs on the average number of channels between the adjacent clusters, denoted by $\beta$. The value of $\beta$ rises as the number of SUs in the network increases. The reason of increase in $\beta$ is that more SUs joining the network lead to

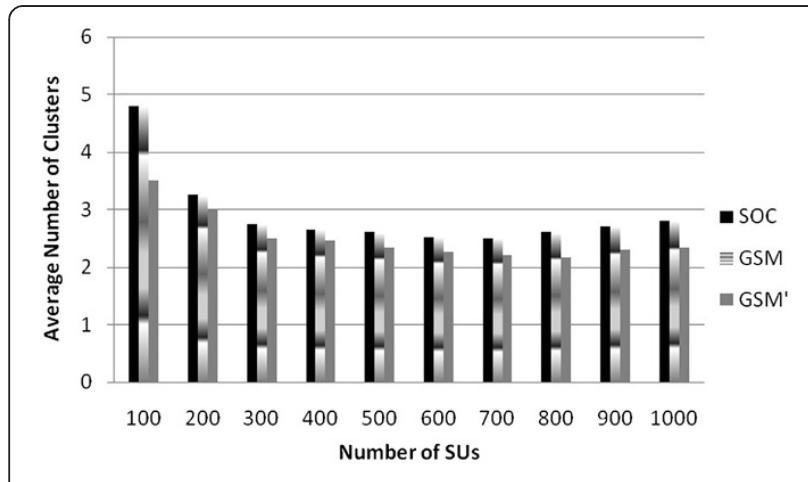

Figure 3 Impact of SU density on the average number of clusters.

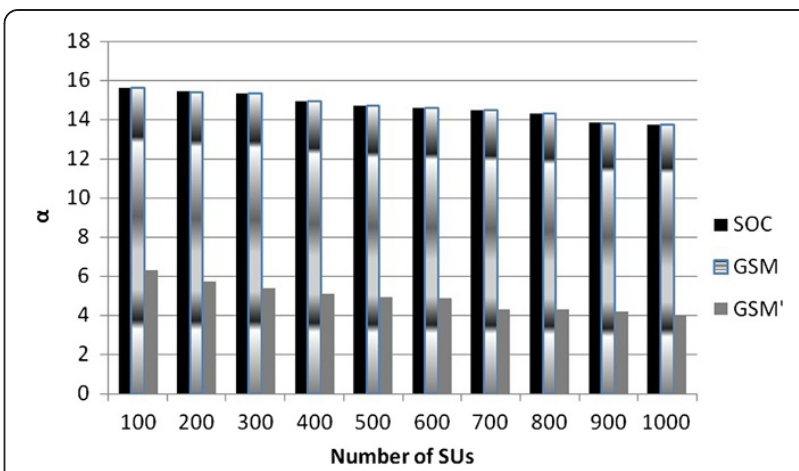

Figure 4 Impact of number of SUs on average number of intra-cluster control channels per cluster $(a)$.

an increase in candidate nodes that can act as gateway nodes between the adjacent clusters. Therefore, choosing a gateway node from a large number of candidates increases the probability of finding a node with a large number of common channels with the control channels of the adjacent clusters. It can be seen that the proposed GSM approach outperforms SOC in terms of $\beta$. However, GSM' performance is comparable with SOC, but lower than GSM because it has less number of common channels designated for intra-cluster communication, which also results in the reduction of degree of the candidate gateway nodes, which is based on the number of channels which a node can have common with the common channel designated in the neighboring cluster. However, the performance of GSM' is comparable with SOC because instead of choosing a gateway node randomly, it selects the node with maximum value of degree and secondly, as it results in large cluster size; hence, more possible candidates for gateway selection are there.

\subsection{Effect of variation in PU density}

Figure 6 shows the impact of the number of PUs on $\alpha$. In this case, the number of PUs is varied from 10 to 100 .

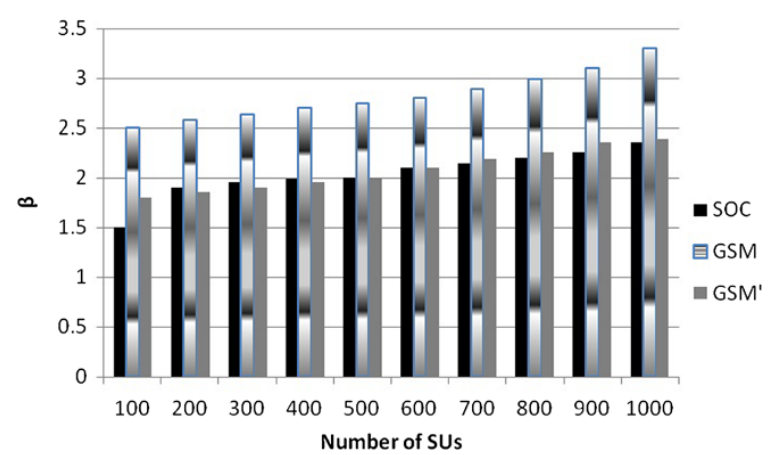

Figure 5 Impact of number of SUs on average number of inter-cluster control channels $(\beta)$. 


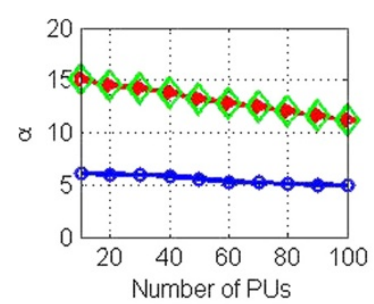

(a)

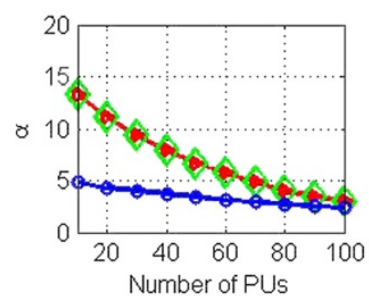

(c)

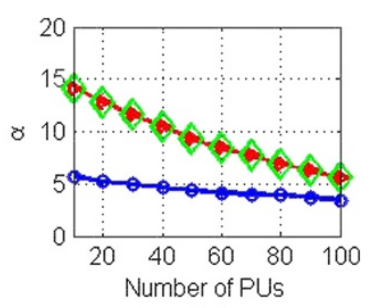

(b)

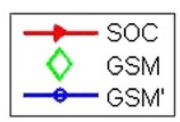

Figure 6 Impact of number of PUs and arrival rate on average number of intra-cluster control channels $(\boldsymbol{a})$. Arrival rate (varying from 0.1 to 0.5 ). (a) $\sigma=0.1$, (b) $\sigma=0.3$, (c) $\sigma=0.5$.

In addition, the simulation is conducted for PU arrival rates from ranges 0.1 to 0.5 . The PU arrival rate $\sigma$ is defined as the frequency with which the PU occupies an available channel. Both GSM and SOC gave the same results. However, GSM' has lower $\alpha$ because it tries to maximize the number of nodes with at least

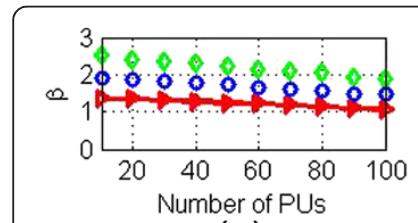

(a)

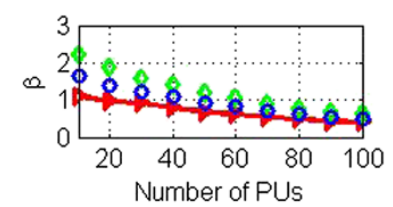

(c)

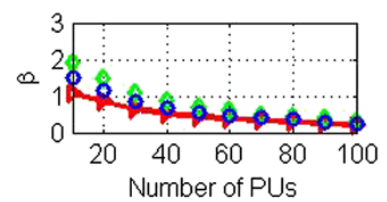

(e)

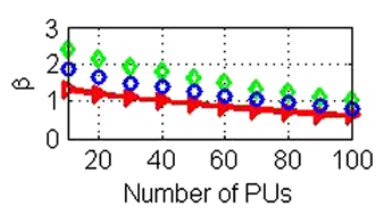

(b)

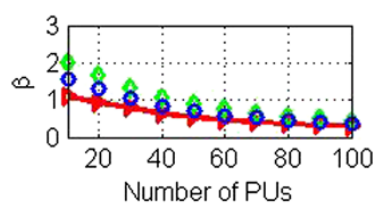

(d)

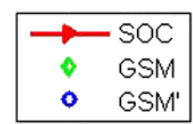

Figure 7 Impact of number of PUs on average number of intercluster control channels with different arrival rates. (a) $\sigma=0.1$, (b) $\sigma=0.3$, (c) $\sigma=0.5$, (d) $\sigma=0.7$, and (e) $\sigma=0.9$. two common channels in a cluster. The value of $\alpha$ decreased as the number of PUs, as well as the PU arrival rate, increased. For example, for GSM, it can be seen that for a PU arrival rate of $0.1, \alpha$ is near 15 when there are 10 PUs, decreases as the number of PUs increases, and finally, reaches a value of 11 when the number of PU increases to 100 .

Figure 7 shows the impact of the number of PUs from 10 to 100 on $\beta$. The simulation is conducted for multiple values of $\mathrm{PU}$ arrival rate varying from 0.1 to 0.9 . The value of $\beta$ decreases as the number of PUs or PU arrival rate increases. It can be seen that GSM outperforms the SOC in terms of $\beta$. GSM' performance is lower than GSM because it has less number of common channels designated for each cluster than GSM but GSM' has higher or similar performance to SOC because SOC employs a random gateway node selection mechanism whereas GSM selects a node with the highest degree among the possible gateway candidate nodes in a cluster. For example, in Figure 7a, for a PU arrival rate of $\sigma=0.1$ and 10 PUs, our proposed scheme has the value of $\beta$ as 2.5 whereas SOC has a value of 1.5 . Hence, our technique is more robust and reliable for inter-cluster coordination than SOC.

\section{Conclusion}

In this study, we examined the control channel establishment problem in CRAHNs. More specifically, we looked at the effect of spectrum heterogeneity and PU dynamic force in the formation of a group-based control channel assignment for CRAHNs. To avoid frequent reclustering, SOC nodes in such a way that more backup channels are available in each cluster. Whenever a channel gets occupied by a PU, instead of re-clustering, control information exchange migrates from the occupied channel to one of the other backup channels. We extended this concept from the intra-cluster level to the inter-cluster level. We surmised that inter-cluster coordination can be strengthened if more channels are between the adjacent clusters and proposed a technique for gateway node selection that ensures that more channels are available for control channel establishment between adjacent clusters. Our proposed approach provides significant improvement over SOC in terms of the average number of inter-cluster control channels.

\section{Competing interests}

The authors declare that they have no competing interests.

\section{Acknowledgements}

This work was supported by the Ministry of Science, ICT and Future Planning (MSIP), Korea, under the Convergence Information Technology Research Center (C-ITRC) support program (NIPA-2013-H0401-13-1003) supervised by the National IT Industry Promotion Agency (NIPA). It was also supported by the Seoul R\&BD Program (SS110012C0214831) and Special Disaster Emergency R\&D Program from National Emergency Management Agency through Kyungil University (2012-NEMA10-002-01010001-2012). 
Received: 31 March 2012 Accepted: 3 June 2013

Published: 21 June 2013

\section{References}

1. IF Akyildiz, W-Y Lee, KR Chowdhury, CRAHNs: cognitive radio adhoc networks. Adhoc Networks 7(5), 810-836 (2009)

2. BF Lo, IF Akyildiz, AM Al-Dhelaan, Efficient recovery control channel design in cognitive radio adhoc networks. IEEE transaction on vehicular technology 59(9), 4513-4526 (2010)

3. IF Akylidiz, WY Lee, MC Vuran, S Mohanty, NeXt generation/dynamic spectrum access/cognitive radio wireless networks: a survey. Computer Networks 50(13), 2127-2159 (2006)

4. BF Lo, A survey of common control channel design in cognitive radio networks. Physical Communication 4, 26-39 (2011)

5. T Chen, H Zhang, G Maggio, I Chlamtac, Topology management in CogMesh: a cluster-based cognitive radio mesh network, in Proceedings of IEEE International conference on Communications ICC'O7 (IEEE, Glasgow, 2007), pp. 6516-6521. http://doi.ieeecomputersociety.org/ 10.1109//CC.2007.1078

6. J Jia, Q Zang, XS Shen, HC-MAC, A Hardware-constrained Cognitive MAC for Efficient Spectrum Management. IEEE journal on selected Areas in Communications 26(1), 106-117 (2008)

7. H Nan, T-I Hyon, J Warland, Comparison of Multichannel MAC protocols. IEEE Transactions on Mobile computing 7(1), 50-65 (2008)

8. J Zhao, H Zheng, G-H Yang, Distributed coordination in dynamic spectrum allocation networks, in Proceedings of IEEE International Symposium on New Frontiers in DySPAN'05 (IEEE, Amsterdam, 2005), pp. 259-268. http://doi.ieeecomputersociety.org/ 10.1109/DYSPAN.2005.1542642

9. N Theis, R Thomas, L DaSilva, Rendezvous for cognitive radios. IEEE Transactions on Mobile Computing 10(2), 216-227 (2011)

10. T Chen, H Zhang, G Maggio, I Chlamtac, CogMesh: a cluster-based cognitive radio network, in Proceedings of IEEE International Symposium on New Frontiers in DySPAN'07 (IEEE, Amsterdam, 2007), pp. 168-178. http://doi. ieeecomputersociety.org/10.1109/DYSPAN.2007.29

11. P Bahl, R Chandra, T Moscibroda, R Murty, M Welsh, White space networking with Wi-Fi like connectivity, in Proceedings of the ACM SIGCOMM 2009 Conference on Data Communication, vol. 39 (ACM, New York, 2009), pp. 27-38

12. S Liu, L Lazos, M Krunz, Cluster-based control channel allocation in opportunistic cognitive radios networks. IEEE Transaction on Mobile Computing (2012). http://doi.ieeecomputersociety.org/10.1109/TMC.2012.33

13. J Zhao, H Zheng, G-H Yang, Spectrum sharing through distributed coordination in dynamic spectrum access networks. Wireless Communication and Mobile computing 7(9), 1061-1075 (2007)

14. T Yucek, H Arslan, A survey of spectrum sensing algorithms for cognitive radio applications. IEEE Communications Surveys Tutorials 11(1), 116-130 (2009)

doi:10.1186/1687-1499-2013-171

Cite this article as: ul Hasan et al:: GSM: gateway selection mechanism for strengthening inter-cluster coordination in cognitive radio ad hoc networks. EURASIP Journal on Wireless Communications and Networking 2013 2013:171

\section{Submit your manuscript to a SpringerOpen ${ }^{\circ}$ journal and benefit from:}

- Convenient online submission

- Rigorous peer review

- Immediate publication on acceptance

- Open access: articles freely available online

- High visibility within the field

- Retaining the copyright to your article

Submit your next manuscript at $\gg$ springeropen.com 